Review

\title{
The visible touch: in planta visualization of protein-protein interactions by fluorophore-based methods Riyaz A Bhat ${ }^{1}$, Thomas Lahaye ${ }^{2}$ and Ralph Panstruga*1
}

\author{
Address: ${ }^{1}$ Department of Plant-Microbe Interactions, Max-Planck-Institute for Plant Breeding Research, Carl-von-Linné-Weg 10, D-50829 Köln, \\ Germany and 2Institute of Genetics, Martin-Luther University, Weinbergweg 10, D-06099 Halle, Germany \\ Email: Riyaz A Bhat - bhat@mpiz-koeln.mpg.de; Thomas Lahaye - lahaye@genetik.uni-halle.de; Ralph Panstruga* - panstrug@mpiz- \\ koeln.mpg.de \\ * Corresponding author
}

Published: 26 June 2006

Plant Methods 2006, 2:12 doi:10.1186/1746-48II-2-12
Received: 28 April 2006

Accepted: 26 June 2006

This article is available from: http://www.plantmethods.com/content/2/1//2

(C) 2006 Bhat et al; licensee BioMed Central Ltd.

This is an Open Access article distributed under the terms of the Creative Commons Attribution License (http://creativecommons.org/licenses/by/2.0), which permits unrestricted use, distribution, and reproduction in any medium, provided the original work is properly cited.

\begin{abstract}
Non-invasive fluorophore-based protein interaction assays like fluorescence resonance energy transfer (FRET) and bimolecular fluorescence complementation (BiFC, also referred to as "split YFP") have been proven invaluable tools to study protein-protein interactions in living cells. Both methods are now frequently used in the plant sciences and are likely to develop into standard techniques for the identification, verification and in-depth analysis of polypeptide interactions. In this review, we address the individual strengths and weaknesses of both approaches and provide an outlook about new directions and possible future developments for both techniques.
\end{abstract}

\section{Background}

Having the first completed plant genomes of the monocotyledonous and dicotyledonous reference species rice (Oryza sativa) and thale cress (Arabidopsis thaliana) in hand [1-3], the analysis of protein function(s) represents a major scientific challenge of the post-genomic era. Many researchers who identified key components of various biological processes by forward or reverse genetic approaches in the past now face a possibly harder task to assign (a) biochemical role(s) to their favorite protein(s). State-of-the-art studies to address this pivotal question frequently involve the analysis of protein-protein interactions to gain insights about the potential cellular function(s) of a protein of interest (POI). Traditionally, the yeast two-hybrid approach represents the method of choice to unravel protein interaction partners of POIs on a large scale and in an unbiased manner [4]. However, since yeast two-hybrid screens are well known to produce false-positive results, subsequent verification of individual interaction partners by further, preferentially in planta, approaches is generally desired. Co-immunoprecipiatation ("pull-down"; [5]), in vitro association studies (e.g. gel overlay assays or "far Western blots" [6], surface plasmon resonance spectroscopy [7]), blue native gel electrophoresis [8], bioluminescence resonance energy transfer (BRET) [9] and fluorescent protein-based methods [1012] are nowadays commonly used to achieve this goal. In this review, we focus on the latter, non-invasive, microscopy-based approaches with a particular emphasis on fluorescence resonance energy transfer (FRET) and bimolecular fluorescence complementation (BiFC) both of which allow monitoring protein-protein interactions in vivo and in real time. Though only recently introduced to the plant sciences, both microscopic techniques have been rapidly absorbed by the community of plant scientists. Given the rapid pace of newly emerging fluorophores with ever improved biophysical properties [11], FRET and BiFC are likely to become even more valuable and common tools in the near future. 


\section{Getting started: general considerations for FRET and BiFC studies}

Before starting any fluorophore-based in planta proteinprotein interaction assays, one should take some general considerations into account. First, it should be noted that all fluorophore-based methods require tagged variants of the POIs, modifications that may alter their physiological parameters. Thus, wherever possible, fluorophore-tagged POIs should be tested for bona fide subcellular localization, stability, and biological activity. The latter can for example be achieved by complementation of mutant phenotypes or, alternatively, by determining protein activities in in vitro assays. Since, conventionally, POIs can be tagged either $\mathrm{N}$ - or C-terminally, and since the site of tagging may determine experimental success in an empirical manner, all possible pair wise combinations should be tested when performing FRET or BiFC assays. Unfavorable circumstances, e.g. terminal targeting signals or transmembrane domains, may however preclude some of these theoretically possible combinations. It should be mentioned that, in principle, some POIs might also be tagged internally [13].

A second aspect that needs to be considered is the expression level of the tagged POIs. Frequently, expression is driven by strong constitutive promoters (e.g., the cauliflower mosaic virus [CaMV] 35S promoter) that may result in ectopic expression and/or overexpression. This might subsequently result in artifacts that may possibly either promote or inhibit particular protein-protein interactions. Thus, wherever possible, the native gene promoters should be used for driving the expression of fluorophore-tagged POIs. It should be stressed, however, that due to the method of gene transfer (e.g. particle bombardment, Agroinfiltration) even constructs with own promoters can result in overexpression when multiple gene copies are transferred into single target cells.

The target species and tissue for transgene expression should also be carefully selected. Ideally, the fluorophoretagged POIs should be expressed in the homologous plant species and in a tissue type that is of biological relevance for the POIs and/or the anticipated protein-protein interaction. Wherever possible, expression should take place in respective (double) null mutants, since endogenous, untagged copies of the POIs may interfere with the protein-protein interaction assay, e.g. by out competing interaction partners. Lines homozygous for T-DNA insertions in the genes encoding both interaction partners represent thus suitable genetic backgrounds for in planta interaction assays. Since it is usually difficult to meet all the criteria mentioned above, one should at least attempt to fulfill as many as possible. In the ideal scenario, however, transgenic lines expressing both fluorophore-tagged POIs under control of their own promoters in a respective double mutant genetic background would be used.

Finally, we would like to stress that both FRET and BiFC represent methods that determine "only" the close physical proximity of two fluorophore-tagged fusion proteins in vivo. It might be debatable whether such a tight contact is the final proof of a true protein-protein interaction or, alternatively, represents merely an indicator of close vicinity, as for example, the co-localization of two polypeptides in a small plasma membrane microdomain (e.g. [14]) or co-presence of two POIs in a large multi-protein complex. Convincing evidence for a direct as opposed to an indirect interaction currently requires in vitro assays using purified recombinant proteins, e.g. the above-mentioned "far Western blots" [6] or surface plasmon resonance spectroscopy [7]. In our view, biologically significant protein-protein interactions are in addition characterized by the involvement of essential amino acid residues in the contact zones of both interaction partners. Mutant variants that are affected in these critical residues and that result in loss of the interaction coincident with an altered plant phenotype are therefore suitable controls to verify the biological significance of a protein-protein interaction. Such mutant variants may originate from genetic screens in planta, might be predicted based on educated guesses or structural data, or could be obtained from yeast-based high-throughput loss-of-interaction assays.

\section{The basic principle of FRET}

Förster (or Fluorescence) Resonance Energy Transfer (FRET) is a biophysical phenomenon that was originally discovered more than half a century ago [15]. Its occurrence is based on a long-range dipole-dipole resonance interaction in which non-radiative energy is transferred from a chromophore in an electronic excited state serving as a "donor", to another molecule (fluorescent or otherwise) serving as the "acceptor". This energy transfer leads to a reduction in the donor's fluorescence intensity and a decreased lifetime in the excited state. If the acceptor molecule is likewise a fluorophore, then FRET additionally gets manifested in the form of an increase in the acceptor's emission intensity.

The efficiency of energy transfer (E) is inversely proportional to the sixth power of the distance between the donor and the acceptor $[15,16]$ :

$E=1 /\left\{1+\left(R / R_{o}\right)^{6}\right\}$

where $R_{o}$ is the distance at which half of the energy is transferred from the donor to the acceptor. $\mathrm{R}_{0}$ is typically between $20-60 \mathrm{~A}^{\circ}(2-6 \mathrm{~nm})$ and thus in the range of conventional protein dimensions. The exact value of $\mathrm{R}_{0}$ is a function of the spectral overlap between donor emission 


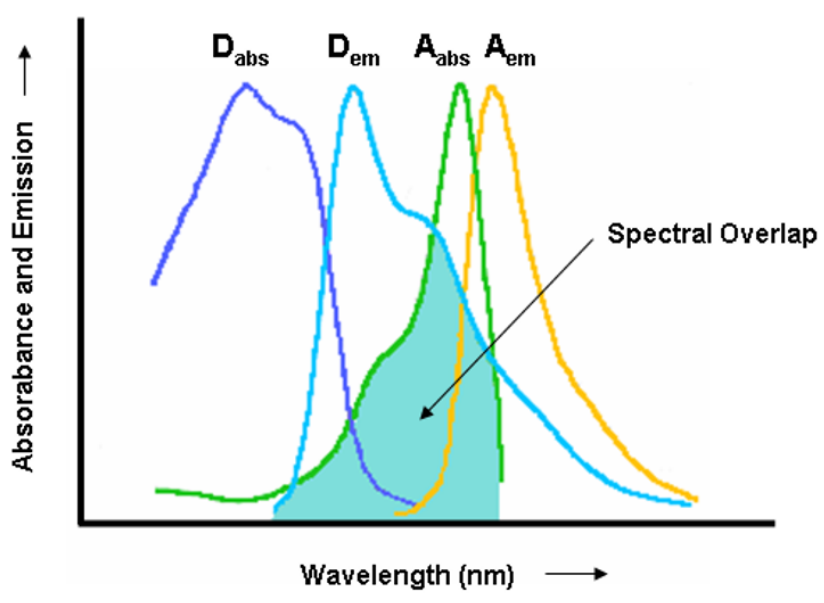

Figure I

Excitation and emission spectra of a commonly used FRET pair. The scheme depicts simplified absorbance and emission spectra of CFP (cyan fluorescent protein; donor; D) and YFP (yellow fluorescent protein; acceptor, A). Overlap between CFP emission and YFP absorption (shaded region) is a prerequisite for FRET. $D_{\text {abs }}$ - Donor absorbance; $D_{\text {em }}-$ Donor emission; $A_{\text {abs }}-$ Acceptor absorbance; $A_{e m}-$ Acceptor emission.

and acceptor excitation spectra (Figure 1), the quantum yield of the donor in the absence of the acceptor, and the relative orientation and rotational freedom of donor and acceptor chromophore transition dipoles. FRET is unique as it is based on molecular interactions in the 1-10 nm range that are sensitive to molecular conformation, association, and separation and thus represents one of the few tools available for measuring nanometer scale distances or changes in such distances $[16,17]$.

\section{FRET as a sensor of protein-protein interactions in living cells}

The availability of genetically encoded fluorophores (green fluorescent protein, GFP; $[18,19]$ and subsequent development of GFP derivatives with suitable spectral properties for FRET (described in [20]) enabled the convenient employment of the FRET principle to address questions in biological systems. In living cells, FRET can occur when protein domains fused to suitable donor and acceptor fluorescent dyes physically interact, i.e. the fluorophores come in close spatial proximity $([10,12]$; Figure 2 ). Such interactions, e.g. between protein domains, can either occur intermolecularly or intramolecularly. Hence, FRET can principally be used to detect either bimolecular protein-protein interactions or conformational alterations within a single polypeptide. In the case of studying an intermolecular interaction, two separate fusion proteins - one containing a donor fluorophore and the other, its putative interacting partner, containing an acceptor fluorophore - are co-expressed in the cell type of choice. If intermolecular FRET is detected this provides direct proof of close proximity of the two chromophores and consequently evidence for the existence of the proteinprotein interaction (Figure 2). Alternatively, for analysis of an intramolecular interaction, fluorophores are fused to different sites (frequently the termini) within a single polypeptide. In this case, relative changes in the FRET intensity are indicative of conformational changes within the test protein, e.g. due to ligand binding, maturation, proteolytic processing etc.

During the past few years, FRET has been extensively used to study protein-protein interactions in a diverse range of organisms and cell types, including yeast [21], animal (e.g. [22,23]) and plant cells [24-37]. Likewise, intracellular sensors based on intramolecular FRET gained increasing attention and are now routinely used as nanosensors to report various intracellular changes of metabolites, e.g. alterations in calcium levels [38] or carbohydrate concentrations [39]. However, despite the widespread interest in detecting protein-protein interactions using FRET microscopy, in the plant sciences reports of successful FRET are still limited in number (Table 1).

Although an inherently extremely inefficient process, recent advances have led to quantitative and qualitative improvements in the FRET technique including increased spatial resolution, distance range and sensitivity [40]. A major problem, however, that remains is achieving FRET in the first instance, because a successful FRET readout requires that the donor and acceptor fluorophores come into close proximity. This can be a limiting factor, especially in the case of large interaction partners (please note that FRET efficiency is inversely correlated with the sixth power of the distance between donor and acceptor fluorophores; see above; [41]). Sterical orientation of the fluorophores in the fusion proteins is another critical and possibly limiting factor [41]. Both fluorophore distance and orientation represent parameters that are difficult to control, except by the empirical insertion of "spacer" sequences between POI and the respective fluorophore. The length and/or amino acid sequence of such spacer sequences have been shown to either positively or negatively influence inter- and intramolecular FRET efficiencies [42-45].

\section{Measuring FRET: being spoilt for choice}

Upon transfer and expression of suitable transgene pairs into target cells, FRET can be measured by several techniques that differ in the precision of data acquisition as well as the complexity of the required instrumentation (online supplement of reference [46]). A decrease in the donor fluorescence intensity (or the quantum yield) and its excited state lifetime, with a corresponding increase in 


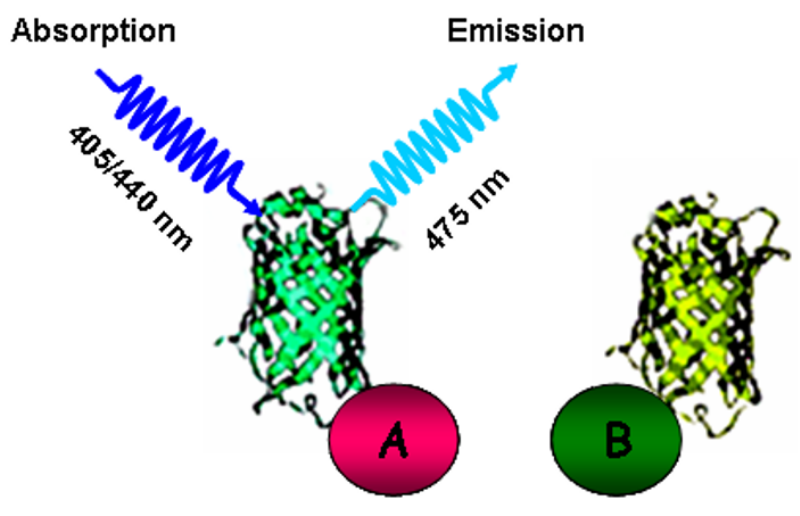

Before FRET

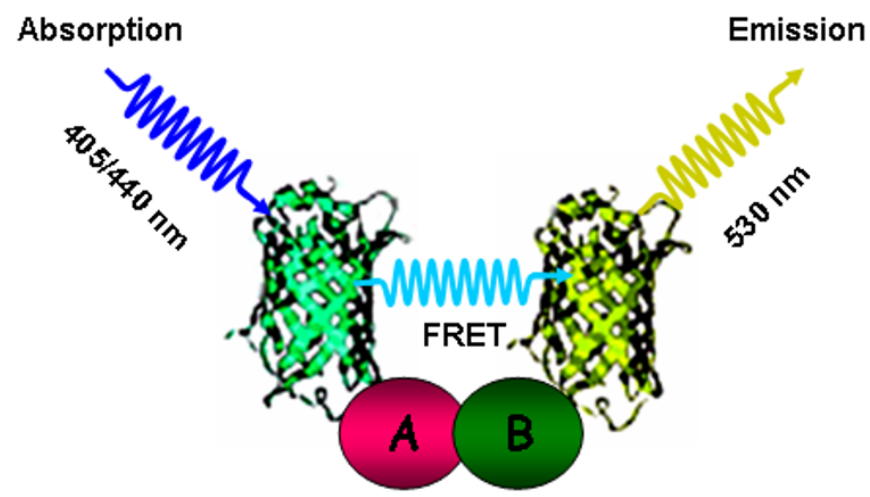

After FRET

Figure 2

Detection of protein-protein interactions via FRET. FRET between cyan fluorescent protein (CFP) as a donor fused to protein A and yellow fluorescent protein (YFP) fused as an acceptor to protein B. Under favorable spatial and angular conditions, interaction between $A$ and $B$ causes a decrease in the intensity of donor (CFP) fluorescence concomitant with an increase in acceptor (YFP) fluorescence. CFP and YFP are depicted as cyan and yellow ribbon models fused to putative interacting proteins $A$ and $B$, respectively.

the acceptor fluorescence intensity (if the acceptor is fluorescent) are the photophysical consequences of FRET. Accordingly, methods for measuring FRET and hence intra- or intermolecular interactions rely on assessing one or more of the above photophysical consequences. Documentation of FRET can be either achieved by rather simple methods like channel FRET or fluorescence spectral imaging microscopy (FSPIM), or by advanced technologies like donor fluorescence recovery after photobleaching (DFRAP) or fluorescence lifetime imaging (FLIM).

Conventionally, FRET was determined by comparing the donor intensity of the donor-acceptor sample to that of the donor only sample, while concurrently comparing the acceptor intensity of the donor-acceptor sample to that of the acceptor only sample (e.g., [24,27,31,34]; Table 1). This method, also known as sensitized emission or channel FRET, requires matching (equimolar) concentrations of fluororohores in the different samples which, being dependent on the cellular expression levels of the proteins under study, is difficult to achieve in an accurate manner. Besides, the direct excitation of the acceptor fluorophore at donor excitation wavelengths requires the subtraction of cross talks and false FRET values using several instrumental correction factors [47]. Additionally, for plant cells, it was reported that the chlorophyll pigments might absorb part of the donor fluorescence and thus lead to false FRET values [48]. Though different mathematical corrections of sophisticated complexity have been designed to rectify these problems $[47,49,50]$, this approach has become less popular due to the development of more reliable FRET techniques (see below).
Fluorescence spectral imaging microscopy (FSPIM) represents a different procedure to document FRET. The method uses a spectroscopic rather than an image-based approach to quantify changes in the acceptor intensity at the donor excitation wavelength. This is achieved by recording emission spectra of the acceptor molecule in the absence or presence of the donor. In comparison to channel FRET, this approach is less sensitive to background noise since spectral rather than intensity information is used as readout. However, as in the case of sensitized emission (see above), a prerequisite is the expression of equal (equimolar) concentrations of the fluorophores, a condition that might be difficult to obtain. Despite this obstacle, the FSPIM procedure has been previously used in various plant FRET studies $[25,26,28,30]$ (Table 1).

A further improvement to measure FRET is DFRAP, donor fluorescence recovery after photobleaching [51]. This procedure is based on the fact that energy transfer from the donor to the acceptor fluorophore will be disrupted upon the irreversible photochemical damage of the acceptor by photobleaching (Figure 3a). As a consequence, the donor fluorescence emission will increase over a short period of time until the acceptor becomes available again (by diffusion from other areas of the live cell) and FRET is re-established (Figure 3b). Since donor fluorescence usually remains unaltered or even decreases after bleaching in the absence of FRET ([51]; Figure 3c), an increase in donor fluorescence is considered a reliable indicator of successful energy transfer. Furthermore, an increase in donor intensity can not be attributed to acceptor bleed through, because the acceptor is not available anymore due to pho- 
Table I: Examples of plant protein-protein interactions studied via FRET or BiFC.

\begin{tabular}{|c|c|c|c|c|}
\hline $\begin{array}{l}\text { Protein-protein } \\
\text { interaction }\end{array}$ & Applied technique & Cell type & Gene transfer method & reference \\
\hline $\begin{array}{l}\text { Phytochrome B- } \\
\text { Cryptochrome } 2\end{array}$ & FRET (channel and DFRAP) & Tobacco protoplasts & Protoplast transfection & {$[24]$} \\
\hline SERK-I (homodimerization) & FRET (FSPIM) & Cowpea mesophyll protoplasts & Protoplast transfection & {$[25]$} \\
\hline $\begin{array}{l}\text { Floral binding protein } 2 \\
\text { (homodimerization) Floral } \\
\text { binding protein II }\end{array}$ & FRET (FSPIM and FLIM) & Petunia leaf protoplasts & Protoplast transfection & [26] \\
\hline TGA5 (homodimerization) & FRET (channel) & Tobacco leaf cells & Agroinfiltration & {$[27]$} \\
\hline SERKI-KAPP & FRET (FSPIM) & Cowpea mesophyll protoplasts & Protoplast transfection & {$[28]$} \\
\hline Opaque2-CGN5/ADA2 & FRET (DFRAP and FLIM) & Cowpea mesophyll protoplasts & Protoplast transfection & {$[29]$} \\
\hline $\begin{array}{l}\text { Lipidated YFP and CFP } \\
\text { variants }\end{array}$ & FRET (FSPIM and FLIM) & Cowpea protoplasts & Protolplast transfection & [30] \\
\hline $\begin{array}{l}\text { AtMinDI } \\
\text { (homodimerization) }\end{array}$ & FRET (channel and DFRAP) & Tobacco leaf epidermal cells & Particle bombardment & {$[31]$} \\
\hline MLO-calmodulin & FRET (DFRAP and FLIM) & Barley leaf epidermal cells & Particle bombardment & {$[32]$} \\
\hline MLO (homodimerization) & FRET (DFRAP) & Barley leaf epidermal cells & Particle bombardment & [33] \\
\hline Vacuolar ATPase subunits & FRET (channel) & Arabidopsis leaf mesophyll protoplasts & Protoplast transfection & [34] \\
\hline MADS box proteins & FRET (FLIM) & Cowpea and Petunia leaf protoplasts & Protoplast transfection & [35] \\
\hline SAGI0I-EDSI & FRET (DFRAP) & Arabidopsis leaf epidermal cells & Particle bombardment & [36] \\
\hline $\begin{array}{l}\text { AtMinEI-AtMinDI, } \\
\text { AtFtsZI-I-AtFtsZ2-I, } \\
\text { AtFtsZ2-I-ARC6 }\end{array}$ & FRET (method unknown) & Tobacco leaf epidermal cells & Particle bombardment & [37] \\
\hline bZIP63 (homodimerization) & $\mathrm{BiFC}$ & Tobacco leaf epidermal cells & Agro-infiltration & {$[68]$} \\
\hline LSDI (homodimerization) & $\mathrm{BiFC}$ & Arabidopsis leaf epidermal cells & Agro-infiltration & {$[68]$} \\
\hline 14-3-3 (homodimerization) & $\mathrm{BiFC}$ & $\begin{array}{l}\text { Arabidopsis cell culture protoplasts and } \\
\text { tobacco leaf epidermal cells }\end{array}$ & $\begin{array}{l}\text { Protoplast transfection } \\
\text { and Agro-infiltration }\end{array}$ & {$[68]$} \\
\hline PFT $\alpha-$ PFT $\beta$ & $\mathrm{BiFC}$ & Arabidopsis leaf epidermal cells & Agro-infiltration & [69] \\
\hline FIE-MEA & $\mathrm{BiFC}$ & $\begin{array}{l}\text { Tobacco and Arabidopsis leaf epidermal } \\
\text { cells }\end{array}$ & Agro-infiltration & [69] \\
\hline VIPI-VirE2, VIPI-VirF & $\mathrm{BiFC}$ & Tobacco and onion leaf epidermal cells & Particle bombardment & {$[70]$} \\
\hline SAD-GAMYB & $\mathrm{BiFC}$ & Onion leaf epidermal cells & Particle bombardment & {$[71]$} \\
\hline $\begin{array}{l}\text { OFPI (homodimerization), } \\
\text { BLHI (homodimerization), } \\
\text { AtOFPI-AtOFPI }\end{array}$ & $\mathrm{BiFC}$ & Tobacco leaf cells & Agroinfiltration & {$[72]$} \\
\hline VirE2-VirE3 & $\mathrm{BiFC}$ & Tobacco and onion leaf epidermal cells & Particle bombardment & {$[73]$} \\
\hline VIPI-VirE2 & $\mathrm{BiFC}$ & Tobacco leaf epidermal cells & Particle bombardment & [74] \\
\hline VIPI-H2A & $\mathrm{BiFC}$ & Tobacco leaf epidermal cells & Particle bombardment & {$[75]$} \\
\hline EIDI-ASKI & $\mathrm{BiFC}$ & $\begin{array}{l}\text { Mustard seedlings and parsley } \\
\text { protoplasts }\end{array}$ & $\begin{array}{l}\text { Particle bombardment and } \\
\text { protoplast transfection }\end{array}$ & [76] \\
\hline FD-FT & $\mathrm{BiFC}$ & Tobacco leaf epidermal cells & Agro-infiltration & {$[77]$} \\
\hline $\begin{array}{l}\text { AtMinEI-AtMinDI, } \\
\text { AtFtsZI-I-AtFtsZ2-I, } \\
\text { AtFtsZ2-I-ARC6 }\end{array}$ & $\mathrm{BiFC}$ & Tobacco leaf epidermal cells & Particle bombardment & [37] \\
\hline $\begin{array}{l}\text { OsOBFI } \\
\text { (homodimerization), } \\
\text { OsOBFI-LIPI9 }\end{array}$ & $\mathrm{BiFC}$ & Onion bulb epidermal cells & Particle bombardment & {$[78]$} \\
\hline $\begin{array}{l}\text { ATHI-STM, BLH3-STM, } \\
\text { BLH9-STM }\end{array}$ & $\mathrm{BiFC}$ & Leek epidermal cells & Particle bombardment & [79] \\
\hline p6 and TGBp2 topology & $\mathrm{BiFC}$ & Tobacco leaf epidermal cells & $\begin{array}{l}\text { Particle bombardment and } \\
\text { Agro-infiltration }\end{array}$ & {$[80]$} \\
\hline
\end{tabular}


(a)
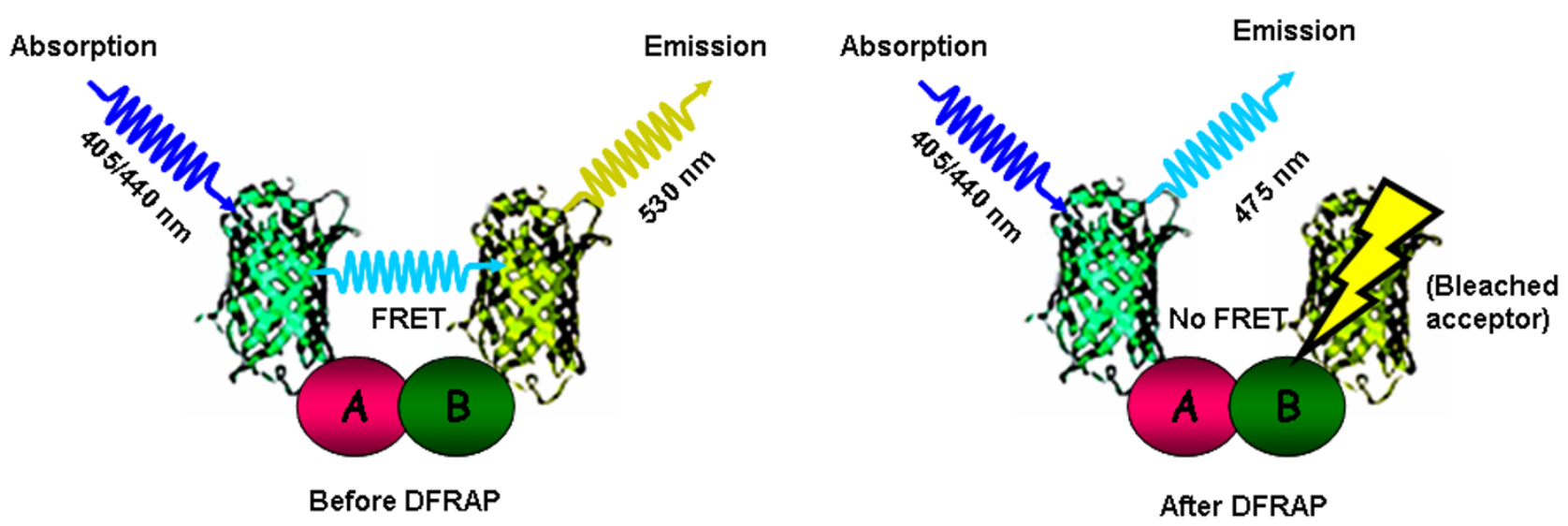

(b)

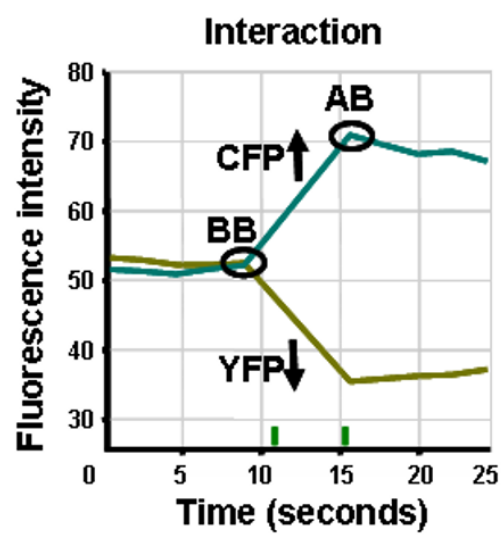

(c)

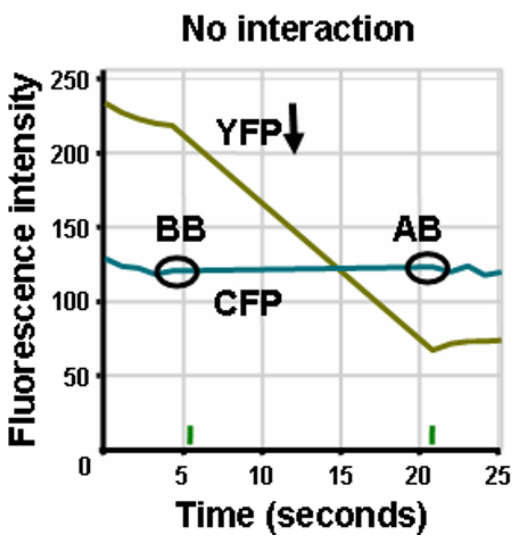

Figure 3

Principle and quantitative assessment of FRET via DFRAP. (a) In case of FRET between the donor CFP and the acceptor YFP due to interaction between two proteins $A$ and $B$, the photochemical destruction of the acceptor abolishes FRET and leads to an increased emission from the donor, CFP. CFP and YFP are depicted as cyan and yellow ribbon models fused to putative interacting proteins $A$ and $B$ respectively. $(\mathbf{b}, \mathbf{c})$. Time-course analysis of fluorescence intensity before and after photobleaching in the presence or absence of a protein-protein interaction. Blue and yellow curves indicate the levels of CFP and YFP fluorescence before and after photobleaching, respectively. In case of FRET, bleaching of the acceptor molecule leads to an increase in donor fluorescence (b). In the absence of interaction between proteins $A$ and $B$, CFP levels before and after the bleach do not vary considerably (c). BB - Before bleach, AB - After bleach.

tobleaching. In contrast to channel FRET or FSPIM, DFRAP is also less sensitive to potential artifacts due to unequal expression levels of the fusion proteins [52]. Owing to the fact that the high mobility of some (e.g. cytoplasmic) polypeptides may accelerate the undesired recovery of FRET, DFRAP measurements should be restricted to a narrow time slot of a few seconds following bleaching (Figure 3). In a range of studies this technique was employed to monitor FRET in various plant systems [24,29,31-33,36] (Table 1). A recent report, however, describes that in DFRAP experiments photoconversion of the bleached YFP into a CFP-like species may occur via an as yet unknown mechanism [52] - an incident that may affect any DFRAP measurements. Although we could not observe such a phenomenon under our experimental conditions (Bhat and Panstruga, unpublished results), this report raises a genuine concern about the employment of DFRAP as a sole FRET sensor.

Finally, the most sophisticated but also a technically demanding way of determining FRET is by measuring the lifetime (the average time that the fluorophore spends in the excited state) of the donor in the presence and absence of the acceptor. This procedure exploits the biophysical fact that FRET leads to a decrease in the donor life-time that can be determined using suitable equipment. Fluorescence life-time imaging microscopy (FLIM) allows the measurement of changes in fluorophore life-times down 


\section{CFP lifetime}

\section{$1.5 \mathrm{~ns} \quad 2.5 \mathrm{~ns}$}

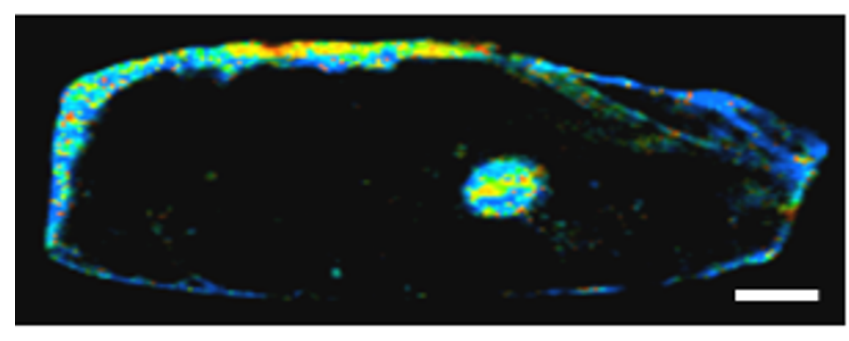

MLO-YFP + CFP-CaM

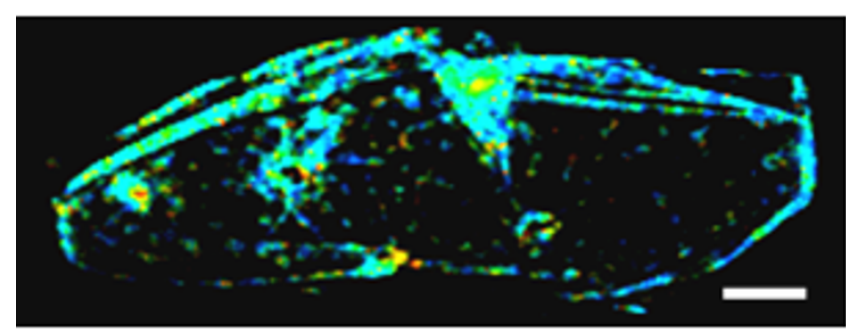

MLO-YFP + CFP-CaM

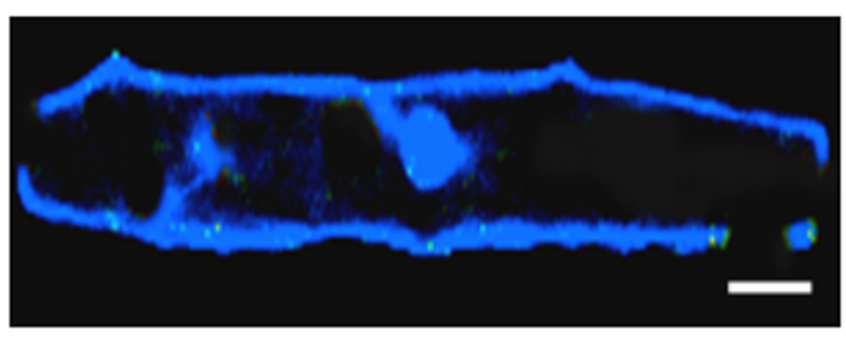

MLO (W423R)-YFP + CFP-CaM

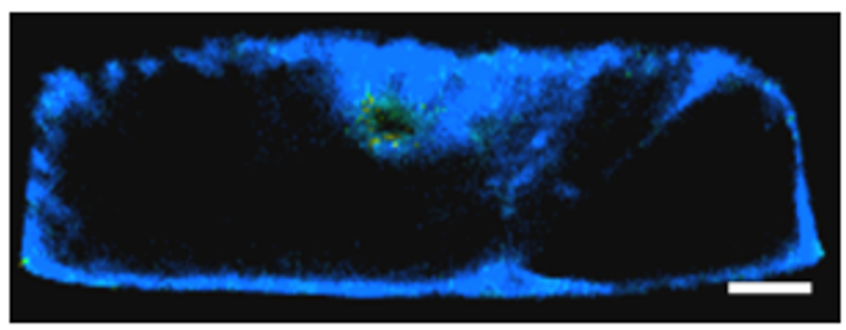

MLO (L420R W423R)-YFP + CFP-CaM

Figure 4

FRET-FLIM analysis of the MLO-calmodulin interaction. Barley MLO is a plant-specific calmodulin-binding protein that functions as a modulator of defence against the common powdery mildew pathogen [90]. YFP-tagged wild-type barley MLO or mutant variants thereof (W423R and L420R W423R, bearing amino acid substitutions in the calmodulin binding domain [90]) were co-expressed with CFP-tagged calmodulin in single barley leaf epidermal cells. FRET-FLIM analysis was performed as described in [32]. Donor fluorophore lifetimes are color-coded according to the scale indicated on top of the Figure. "Warmer" colors are indicative of shorter donor fluorophore lifetimes and thus interaction between MLO and calmodulin. Size bar, $20 \mu \mathrm{m}$.

to pico-second levels [53,54]. FLIM measurements, as opposed to simple intensity or DFRAP measurements, have the advantage of being concentration-independent and also free of interference by spectral cross-talk, photobleaching or absorption of the donor fluorescence by chlorophyll [26]. The latter can become a problem in FSPIM studies or sensitized emission assays when looking at the quenching of the donor or the sensitized emission of the acceptor, respectively. Additionally, if multiple lifetimes can be resolved, FLIM is able to differentiate subpopulations with different amounts of energy transfer [48] and thus provides a quantitative interaction map of a cell with a single measurement (Figure 4).

Several recent studies have used the potential of FLIM to capture interactions between partner proteins playing dif- ferent roles in regulation of transcription, development as well as disease signaling in plants $[26,29,30,32,35]$ (Table 1 ). However, the technique is still far from becoming a routine method for monitoring protein-protein interactions in plants or any other system. Major obstacles are the associated costs and the current limited availability of lifetime systems. Additionally, setting up the FLIM system for determining FRET can be laborious and technically demanding. The commercialization of systems with improved and dedicated image analysis software should increase the popularity of lifetime imaging.

\section{The choice of fluorophores for FRET}

Pairs of fluorophores with overlapping emission and excitation spectra are a prerequisite for FRET (Figure 1). Ideally, the acceptor should exhibit minimal excitation at the 

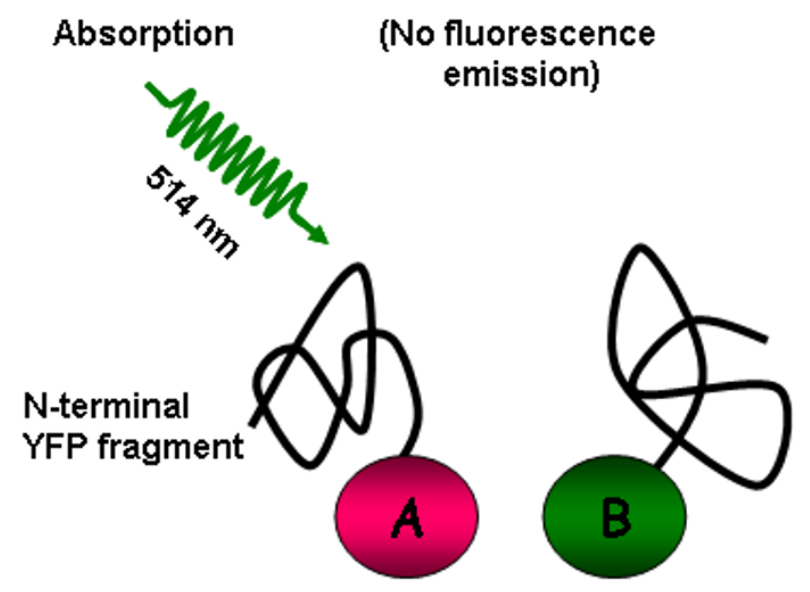

C-terminal YFP fragment

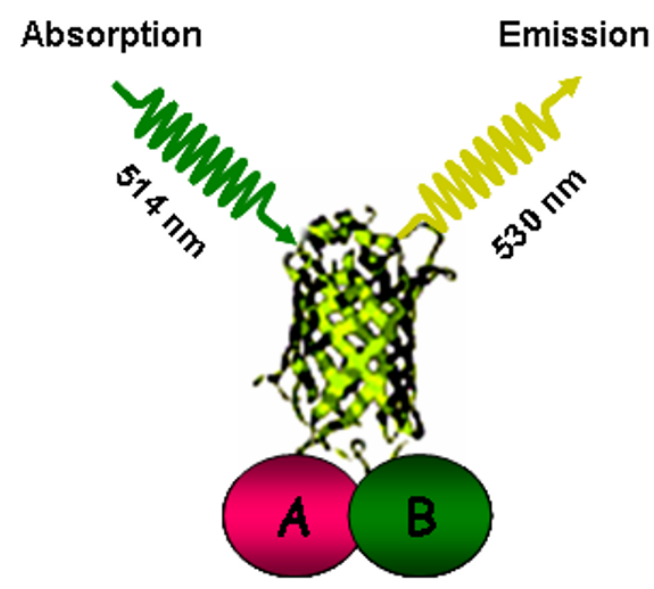

After BiFC

\section{Figure 5}

Principle of the BiFC assay. The scheme depicts the principle of the BiFC assay, exemplified by a split YFP fluorophore. Proteins $A$ and $B$ are fused to $N$ - and $C$-terminal fragments of YFP, respectively. In the absence of an interaction between $A$ and $B$, the fluorophore halves remain non-functional. Following interaction between $A$ and $B$, a functional fluorophore is reconstituted which exhibits emission of fluorescence upon excitation with an appropriate wavelength.

wavelength used to excite the donor fluorophore. Chromophore-mutated green fluorescent proteins (GFPs) with an excellent spectral overlap have been widely used in FRET studies [55]. Initially BFP (blue fluorescent protein) was heralded as an ideal FRET partner with GFP [48]. However, owing to the low photostability of BFP [51], identification of the CFP (Cyan fluorescent protein) and YFP (Yellow fluorescent protein) mutant versions of GFP replaced the BFP-GFP pair as donor-acceptor couple in FRET studies. Since then, the original CFP-YFP FRET pairs or their mutant versions such as monomeric mCFP and mYFP, Cerulean (a brighter CFP), Venus and Citrine (both improved YFPs) or recently identified CyPet and YPet are being extensively used for FRET studies in living cells [5658]. Identification and use of RFP (Red fluorescent protein) as an acceptor to the GFP in FRET experiments was also exploited successfully when Más et al. [24] used GFPRFP as a donor-acceptor FRET pair to analyse the molecular interaction between Arabidopsis phytoreceptors PHY$\mathrm{B}$ and CRY2. Recently described mutations in RFP to produce fluorescent proteins over the whole visible spectrum, e.g mOrange, mPlum, mCherry etc. [56,59], have opened up the possibility of using these as acceptors with GFPs like T-Sapphire (an improved GFP with a single excitation peak and a huge Stoke's shift; excitation wavelength 399 $\mathrm{nm}$; emission wavelength $511 \mathrm{~nm}$ ) as donors [60]. Generally, monomeric fluorophore versions should be used [61] to minimize the reported low-affinity oligomerization of GFP variants that may affect FRET measurements [62]. A comprehensive review article about the choice of fluorophores, including FRET studies, has been recently published and is referred to for further details on this topic

[56].

\section{Bimolecular fluorescence complementation (BiFC)}

The BiFC (also known as "split YFP") assay is based on the observation that $\mathrm{N}$ - and C-terminal sub fragments of GFP (or derivatives thereof, e.g. YFP) do not spontaneously reconstitute a functional fluorophore. However, if fused to interacting proteins, the two non-functional halves of the fluorophore are brought into tight contact, refold together and generate de novo fluorescence. Thus, by BiFC, the interaction status of two POIs can be easily monitored via fluorescence emission upon excitation with a suitable wavelength (Figures 5 and 6).

As many other interaction reporter systems, the BiFC assay is a fragment complementation assay (FCA). GFP and its variants are especially attractive reporters for FCA-based interaction studies, because i) no exogenous reagent needs to be added to detect the reassembled protein and ii) GFP and its derivatives are known to express, fold, mature and fluoresce in virtually every cell type and subcellular structure in which they have been tested. Mutational studies uncovered permissive sites within the GFP molecule that allow insertions without disturbing GFP fluorescence $[63,64]$ and thus paved the way to create a GFP-based FCA assay. Subsequently many different split points have been studied for GFP and its derivatives (reviewed in [65]). Ghosh and colleagues [66] were the 


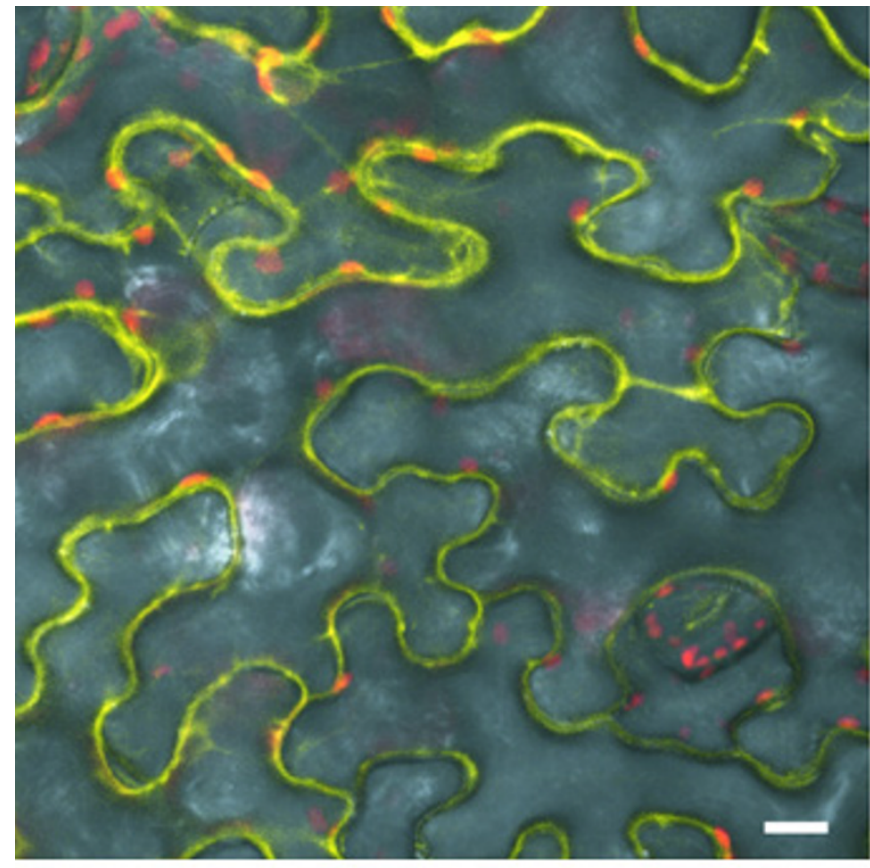

HSPGO-HN + YC-HSP9O

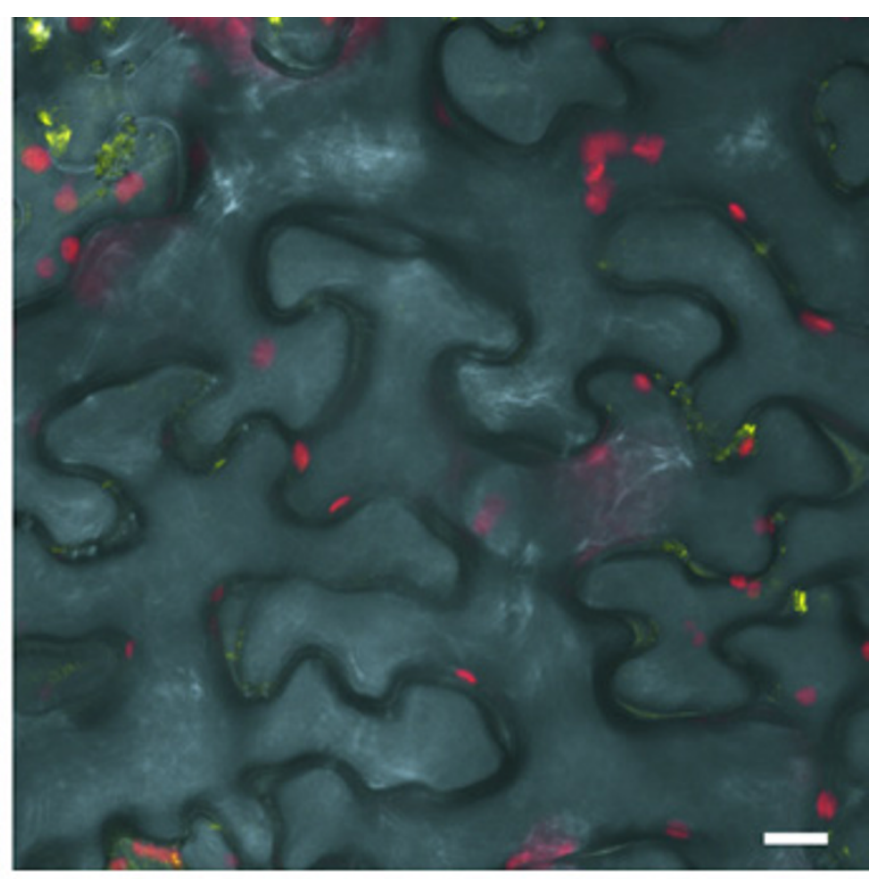

HSPSO-HN + YC-IMP

\section{Figure 6}

Confocal images of bimolecular fluorescence complementation (BiFC) studies. The micrographs show a positive result (HSP90 dimerization; [9I]) as well as a negative result (expected absence of interaction between HSP90 and importin $\alpha$, a mediator of nuclear transportation) of the BiFC assay. HSP90 tagged with the N-terminal fragment of YFP (HSP90-YN) was co-expressed in Nicotiana benthamiana leaves by Agrobacterium tumefaciens transient transformation with the C-terminal fragment of YFP fused to either HSP90 (YC-HSP90; left side) or importin $\alpha$ (YC-IMP; right side). Yellow colour results from the functional complementation of the two halves of the YFP fluorophore and indicates interaction of corresponding fusion proteins. Size bar, $10 \mu \mathrm{m}$.

first to report the use of a split GFP reporter in vitro and in E. coli to study protein-protein interaction. Subsequently, $\mathrm{Hu}$ and co-workers showed that a split fluorophore reporter can also be used in mammalian cells [67]. Finally, Bracha-Dori and colleagues as well as Walter et al. reported on usage of the BiFC system in plant cells $[68,69]$. Meanwhile many more reports on the use of BiFC in planta have been published [70-80] and the system is becoming a routinely used approach to study protein-protein interactions in living plant cells.

When using BiFC for interaction studies one should be aware of the pitfalls and limitations of this technique. One major drawback as well as an advantage of the BiFC approach lies in the irreversibility of complex formation [81]. This obscures the physiological time course of interactions but also traps and accumulates transient and weak associations, thus increasing the sensitivity of detection. An important question in BiFC studies is if the affinity of two interaction partners corresponds to the degree of cellular fluorescence. A recent study shows that BiFC-derived fluorescence does not directly relate to the protein-ligand dissociation constant for an arbitrary protein-ligand pair [81]. However, when studying several interactors for a given protein, BiFC is indeed useful for discriminating strongly bound ligands from weakly bound ones [81]. A further aspect that needs to be considered is the maturation time of the fluorophore tag. Intact (full-size) GFP, for example, requires several hours to mature in the cell [20], and it is conceivable that the intermolecular reconstitution of a split fluorophore may take even longer. Thus, proteins with high turnover rates might not be amenable to BiFC studies. However, modified fast-maturating GFP variants with increased fluorescence intensities have been shown to be suitable for BiFC studies in green monkey kidney fibroblast (COS) cells [82].

Another important aspect is to what extent overexpression may produce artefactual results in BiFC assays. Transfection studies with COS cells revealed that high amounts of vector DNA, containing $\mathrm{N}$ - and C-terminal YFP sensor peptides respectively, provoked unspecific fluorescence 
Table 2: Comparison between BiFC and FRET.

\begin{tabular}{|c|c|c|}
\hline & BiFC & FRET \\
\hline Required microscopic equipment & simple & extensive \\
\hline Data analysis and computation required & - & + \\
\hline Concentration dependence & high & $\begin{array}{l}\text { high (channel FRET, FSPIM) or low (DFRAP, } \\
\text { FLIM) }\end{array}$ \\
\hline Specific problems & $\begin{array}{l}\text { false positives (possibly due to high expression } \\
\text { levels and/or irreversibility) }\end{array}$ & $\begin{array}{l}\text { donor bleed-through (channel FRET, FSPIM), } \\
\text { photoconversion, protein mobility (DFRAP) }\end{array}$ \\
\hline $\begin{array}{l}\text { Endogenous expression control (i.e. } \\
\text { visualization of tagged partners with } \\
\text { subcellular resolution) }\end{array}$ & - & + \\
\hline Monitoring interaction dynamics & - (fluorophore reconstitution irreversible) & + (interactions reversible) \\
\hline Subcellular resolution of interaction sites & high & $\begin{array}{l}\text { high (FLIM) or low (channel FRET, FSPIM or } \\
\text { DFRAP) }\end{array}$ \\
\hline Suitable for tri-molecular interactions & - & $+/-$ \\
\hline $\begin{array}{l}\text { Suitable for monitoring multiple distinct } \\
\text { interaction pairs inside the same cell } \\
\text { ("multicolor") }\end{array}$ & + & - \\
\hline Suitable for medium to high throughput & + & - \\
\hline
\end{tabular}

emission [83]. In contrast low amounts of vector resulted in detectable fluorescence only if interacting proteins were fused to the sensor peptides. These data demonstrate that the level of protein expression in BiFC assays has to be carefully controlled to avoid false positive interactions. It is therefore strongly recommended to perform control experiments employing either "empty" vectors or expressing fusion proteins that are not expected to interact with the POIs. In this context it is also worthwhile mentioning that the commonly used BiFC vectors for in planta expression generally contain the strong constitutive CaMV 35S promoter $[68,69]$. It has been demonstrated that CaMV 35S-expressed C- and $\mathrm{N}$-terminal sensor peptides can produce a certain degree of fluorescence even if they are not fused to interacting proteins. This indicates that complementary sensor peptides are capable of a non-assisted interaction (NAI) [80]. However, NAIs are observed only if the complementary sensor peptides are located in the same subcellular compartment. This highlights the fact that appropriate negative controls in BiFC association studies have to be located in the same compartment as the interaction partners under investigation. Development of novel fluorophore derivatives that can be split in less "sticky" halves, either by protein design or via random mutagenesis, may diminish NAI-associated difficulties in the future.

Although NAIs pose a problem in interaction studies, they are quite useful to determine the subcellular localization of a protein or a protein domain. In NAI-based localization studies, one sensor peptide is fused to the POI while the complementary sensor peptide is fused to a targeting signal for a suspected subcellular destination (e.g. targeting signal for the nucleus, chloroplasts or mitochondria). If the compartment-targeted sensor peptide produces flu- orescence in combination with a protein that is fused to the complementary sensor peptide, one can deduce that the investigated protein localizes to the sensor peptidetargeted compartment. This assay has been recently used to determine the topology of an integral membrane protein [80]. In the future, this approach might become a common procedure to complement GFP-based protein localization studies.

Based on its designation one might expect that BiFC provides a direct measure for bimolecular interactions. However, as for all FCAs that are carried out in vivo, it is possible that a third protein mediates the interaction. In this case the observed interaction would be indirect. It has been estimated that fluorescence complementation can occur when fragments are fused to positions that are separated by a distance of approximately $10 \mathrm{~nm}(100 \AA)$, provided that there is enough flexibility to allow reconstitution of the split YFP fragments [67]. Due to these topological constraints, BiFC will strongly favor the detection of direct protein-protein interactions as opposed to those that occur through complexes. In this context it also needs to be considered that $\mathrm{N}$ - or C-terminal halves of the GFP derivative used can be fused to the $\mathrm{N}$ - or C-terminal part of the POIs, thus resulting in four different combinations that can theoretically be tested. BiFC-based analysis of interacting proteins has revealed that not all possible combinations of the fusion proteins may give rise to identical results [69], suggesting that each POI should be fused with all possible sensor peptide combinations to ensure fidelity of the experimental outcome.

Perhaps the most exciting application of the BiFC reporter system is the possibility of saturating high-throughput in planta interactor screens and thus the replacement of the 
conventional yeast two-hybrid assay. The envisaged BiFCbased interactor screen of cDNA expression libraries has already been carried out in a suspension cell culture of mammalian COS cells [84]. In brief, a cDNA library was fused to the N-terminal half of GFP while the bait protein was fused to the C-terminal half of GFP. After co-transfection of the bait protein and the prey library, flourescing COS cells were collected by fluorescence-activated cell sorting (FACS), an approach that allows spectral analysis and sorting of 1,000-10,000 cells per second. Expression plasmids were extracted from the collected fluorescing cells and clones encoding putatively interacting proteins further enriched in a second round of co-transfection/cell sorting. Inserts of positive clones were subsequently subjected to DNA sequencing. It remains to be seen whether this screen indeed yielded authentic interactors since the identified candidates have not been studied yet by complementary approaches. To date, BiFC studies in plants have been conducted by either particle bombardmentbased or Agrobacterium-mediated transient transformation of plant tissues (Table 1). However, in order to accomplish saturating mass screens, plant scientists need to transfer the BiFC assay to suspension cell culture or protoplast systems. In addition, automated analyses in a microtitre plate format or by the aid of a cell sorter (FACS) will be needed to perform such high-throughput BiFC interactor screens in planta.

\section{Beyond conventional FRET and BiFC: studying multiple interactions simultaneously and analyzing interactions with more than two partners}

Many proteins potentially have a large number of alternative interaction partners in each cell. Some of these interactions might be mutually exclusive, possibly resulting in competition for shared interaction partners. Interactions between alternative partners in living cells can be studied by a multicolor BiFC assay [85]. This assay is based on the use of fragments of fluorescent proteins with distinct spectral characteristics. Bimolecular complexes formed between these fragments can be visualized using different excitation and emission wavelengths, enabling parallel visualization of multiple interactions in the same cell. Systematic analysis of twelve combinations of different GFP, YFP and CFP sub-fragments resulted in the identification of twelve bimolecular fluorescent complexes with seven distinct spectra [85] that provide an ideal basis for multicolor BiFC. This advancement will not only allow monitoring alternative interaction partners of a POI, but also studying multiple pair wise interactions simultaneously inside the same cell. However, the use of this system is limited to laboratories that have sophisticated detection systems that are capable to discriminate between several GFP derivatives with similar excitation and emission spectra. In contrast to BiFC, it appears that due to inevitable crosstalk between the currently available fluorophores,
FRET is confined to a single interaction pair within a particular cell.

Many meaningful biological protein interactions involve polypeptide complexes with more than two interacting proteins. Conventional FRET or BiFC between two components has been unable to shed light on the establishment and/or dynamics of such multi-protein complexes. Recently a three component FRET system based on sensitized emission and DFRAP was described in the context of a three way protein-protein interaction in mammalian cells. ECFP, EYFP and mRFP fused to three different proteins revealed mutually dependent energy transfer between the three fusion proteins in an endosomal compartment [86]. Though careful experimental and theoretical considerations are required to discriminate sequential from parallel energy transfer, this method holds a great promise to characterize three-way interactions during complex signaling processes in plant cells as well.

\section{Comparison between FRET and BiFC}

Both FRET and BiFC generally provide reliable in planta protein-protein interaction data. However, as outlined above in detail, both approaches have their individual advantages and disadvantages (Table 2). This is primarily due to the fact that BiFC is based on a gain of fluorescence, while FRET causes a quantitative change in fluorescence. Since FRET-based studies rely on specific detection of spectrally similar fluorophores or even quantification of fluorophore lifetimes, they require sophisticated, expensive instrumentation while BIFC can be measured by standard epifluorescence microscopy equipment $[46,53]$. Likewise, FRET assays need comprehensive post-imaging data analysis, while this additional step is generally not required for BiFC studies. Since BiFC sensor peptides fluoresce only upon interaction of their fusion partners, it is impossible to visually confirm that both fusion proteins are being made in the absence of an interaction. Thus, in BiFC studies, rather time consuming immunoblot analysis is required to validate expression of the fusion proteins in the absence of interaction. In contrast, FRET sensor peptides are intrinsically fluorescent, which permits detection and quantification of fusion protein levels independently of their interaction status. The irreversibility of the re-established fluorophore complex in BiFC assays is an ambivalent facet: on one hand, this feature enhances sensitivity in determining low-affinity interactions; on the other hand, this attribute may be the cause of false-positive results and also prevents the analysis of dynamic interactions. In this context, false-positive BiFC results may result from high expression levels, while at least some FRET techniques (DFRAP, FLIM) are largely independent of fluorophore concentrations (and thus independent of equimolar and/or physiological expression levels). Finally, BiFC resolves interactions with a high intracellular 
spatial resolution and is additionally suitable for medium- to high-throughput approaches. In conclusion, the choice between BiFC and FRET depends on the available instrumentation, the skills of the researcher and the experimental requirements. Since BiFC and FRET represent complementary experimental approaches and since in both cases false-negative data may result from trivial causes such as fusion protein stability or unfavorable polypeptide conformation, we generally recommend pursuing both techniques whenever possible.

\section{Conclusion and outlook}

During the past few years, FRET and BiFC have been established as reliable techniques for the analysis of proteinprotein interactions in living plant cells. However, one of the obvious disadvantages of both approaches is the fact that they often involve ectopic expression and/or overexpression of the respective fusion proteins (see above and Table 1). This may cause artifacts that could possibly either promote or inhibit particular protein-protein interactions. Thus, fluorophore-based in planta protein-protein interaction assays that operate at low, physiological expression levels or even at the single molecule stage are highly desirable. Dual-color Fluorescence cross correlation spectroscopy (FCCS) represents such a method that is based on single molecule detection. This technique involves recognition of two fluorophore-tagged polypeptide species in a sub-femtoliter measurement volume. The two polypeptides are marked with distinct fluorescent labels that can be separately excited and detected. Coincidence of signal fluctuations of both fluorophores in the detection volume indicates co-migration and thus association of the two proteins at the single molecule level $[87,88]$. Despite the potential power of this method, it has not been extensively applied in vivo to date [88] and we do not know of any application in the plant sciences yet. It remains to be seen whether FCCS (or advances of related techniques [89]) will evolve as the next generation of sophisticated in planta protein-protein interaction assays.

\section{Abbreviations}

BFP Blue Fluorescent Protein

BiFC Bimolecular Fluorescence Complementation

CaMV Cauliflower 쓰osaic Virus

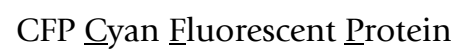

DFRAP Donor Fluorescence Recovery a fter Photobleaching

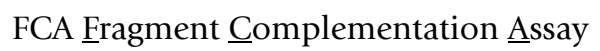

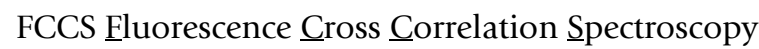

FLIM Fluorescence Lifetime $\underline{\text { Imaging }}$

FRET Fluorescence (Förster) Resonance Energy Transfer

FSPIM Fluorescence Spectral Imaging Microscopy

GFP Green Fluorescent Protein

NAI Non- Assisted Interaction

POI Protein of Interest

RFP $\underline{\text { Red }}$ Fluorescent Protein

YFP Yellow Fluorescent Protein

\section{Competing interests}

The author(s) declare that they have no competing interests.

\section{Authors' contributions}

RAB wrote the draft for the FRET section, TL was the main author for the BiFC part. RAB designed schemes depicted in Figures 1, 2, 3 and 5. RP wrote the Abstract, Introduction and Conclusion sections, copy-edited the manuscript, and suggested Figure layouts. All authors have read and agreed on the final version of the manuscript.

\section{Acknowledgements}

We are grateful to Sebastian Schornack for providing the micrographs shown in Figure 6. We wish to thank Matthew Humphry for critically reading the manuscript. We acknowledge the pop band "Genesis" whose CD entitled "Invisible touch" inspired the title of this paper. Work in the lab of T.L. is in part funded by DFG SFB 648 and DFG grant LA 1338/2-2. Work in the lab of R.P. is supported by grants of the Max-Planck society and DFG grants PA-86I/I and PA-86I/4.

\section{References}

I. The Arabidopsis Genome Initiative: Analysis of the genome sequence of the flowering plant Arabidopsis thaliana. Nature 2000, 408:796-8I5.

2. Goff SA, Ricke D, Lan TH, Presting G, Wang RL, Dunn M, Glazebrook J, Sessions A, Oeller P, Varma H, Hadley D, Hutchinson D, Martin C, Katagiri F, Lange BM, Moughamer T, Xia Y, Budworth P, Zhong JP, Miguel T, Paszkowski U, Zhang SP, Colbert M, Sun WL, Chen LL, Cooper B, Park S, Wood TC, Mao L, Quail P, Wing R, Dean R, Yu YS, Zharkikh A, Shen R, Sahasrabudhe S, Thomas A, Cannings R, Gutin A, Pruss D, Reid J, Tavtigian S, Mitchell J, Eldredge G, Scholl T, Miller RM, Bhatnagar S, Adey N, Rubano T, Tusneem N, Robinson R, Feldhaus J, Macalma T, Oliphant A, Briggs S: A draft sequence of the rice genome (Oryza sativa L. ssp japonica). Science 2002, 296:92-100.

3. Yu J, Hu SN, Wang J, Wong GKS, Li SG, Liu B, Deng YJ, Dai L, Zhou $Y$, Zhang XQ, Cao ML, Liu J, Sun JD, Tang JB, Chen YJ, Huang XB, Lin W, Ye C, Tong W, Cong LJ, Geng JN, Han YJ, Li L, Li W, Hu GQ, Huang XG, Li WJ, Li J, Liu ZW, Liu JP, Qi QH, Liu JS, Li T, Wang XG, Lu H, Wu TT, Zhu M, Ni PX, Han H, Dong W, Ren XY, Feng XL, Cui P, Li XR, Wang H, Xu X, Zhai WX, Xu Z, Zhang JS, He SJ, Zhang JG, Xu JC, Zhang KL, Zheng XW, Dong JH, Zeng WY, Tao L, Ye J, Tan J, Ren XD, Chen XW, He J, Liu DF, Tian W, Tian CG, Xia HG, Bao QY, Li G, Gao H, Cao T, Zhao WM, Li P, Chen W, Wang XD, Zhang Y, Hu JF, Liu S, Yang J, Zhang GY, Xiong YQ, Li ZJ, Mao L, Zhou CS, Zhu Z, Chen RS, Hao BL, Zheng WM, Chen SY, Guo W, Li GJ, Liu SQ, Tao 
M, Zhu LH, Yuan LP, Yang HM: A draft sequence of the rice genome (Oryza sativa L. ssp indica). Science 2002, 296:79-92.

4. Causier B, Davies B: Analysing protein-protein interactions with the yeast two-hybrid system. Plant Mol Biol 2002, 50:855-870.

5. Masters MC: Co-immunoprecipitation from transfected cells. Methods Mol Biol 2004, 261:337-350.

6. Hall RA: Studying protein-protein interactions via blot overlay or Far Western blot. Methods Mol Biol 2004, 26 I: I67-I74.

7. Cooper MA: Label-free screening of bio-molecular interactions. Anal Bioanal Chem 2003, 377:834-842.

8. Eubel H, Braun HP, Millar AH: Blue-native PAGE in plants: a tool in analysis of protein-protein interactions. Plant Methods 2005, I:II.

9. Pfleger KDG, Eidne KA: Illuminating insights into protein-protein interactions using bioluminescence resonance energy transfer (BRET). Nature Methods 2006, 3: I65-174.

10. Hink MA, Bisseling T, Visser A: Imaging protein-protein interactions in living cells. Plant Mol Biol 2002, 50:87I-883.

II. Chapman S, Oparka KJ, Roberts AG: New tools for in vivo fluorescence tagging. Curr Opin Plant Biol 2005, 8:565-573.

12. Dixit $R, C y r R$, Gilroy S: Using intrinsically fluorescent proteins for plant cell imaging. Plant 」 2006, 45:599-6/5.

13. Frank M, Thumer L, Lohse MJ, Bunemann M: G protein activation without subunit dissociation depends on a $\mathbf{G}$ alpha(i)-specific region. J Biol Chem 2005, 280:24584-24590.

14. Sharma P, Varma R, Sarasij RC, Ira , Gousset K, Krishnamoorthy G, Rao M, Mayor S: Nanoscale organization of multiple GPIanchored proteins in living cell membranes. Cell 2004, I 1 6:577-589.

15. Förster T: Zwischenmolekulare Energiewanderung und Fluoreszenz. Annalen der Physik 1948, 2:55-75.

16. Stryer L, Haugland RP: Energy transfer - a spectroscopic ruler. Proc Natl Acad Sci USA 1967, 58:71 9-726.

17. Jares-Erijman EA, Jovin TM: FRET imaging. Nat Biotechnol 2003, 21:1387-1795

18. Prasher DC, Eckenrode VK, Ward WW, Prendergast FG, Cormier MJ: Primary structure of the Aequorea victoria green-fluorescent protein. Gene 1992, I I I:229-233.

19. Chalfie M, Tu Y, Euskirchen G, Ward WW, Prasher DC: Green fluorescent protein as a marker for gene expression. Science 1994, 263:802-805.

20. Tsien RY: The green fluorescent protein. Annu Rev Biochem 1998 , 67:509-544.

21. Muller EGD, Snydsman BE, Novik I, Hailey DW, Gestaut DR, Niemann CA, O'Toole ET, Giddings TH, Sundin BA, Davis TN: The organization of the core proteins of the yeast spindle pole body. Mol Biol Cell 2005, 16:334I-3352.

22. Miyawaki A: Visualization of the spatial and temporal dynamics of intracellular signaling. Dev Cell 2003, 4:295-305.

23. Silvius JR, Nabi IR: Fluorescence-quenching and resonance energy transfer studies of lipid microdomains in model and biological membranes (Review). Mol Membr Biol 2006, 23:5-16.

24. Más P, Devlin PF, Panda S, Kay SA: Functional interaction of phytochrome B and cryptochrome 2. Nature 2000, 408:207-II.

25. Shah K, Gadella TW Jr, van Erp H, Hecht V, de Vries SC: Subcellular localization and oligomerization of the Arabidopsis thaliana somatic embryogenesis receptor kinase I protein. J Mol Biol 200I, 309:64I-55.

26. Immink RGH, Gadella TWJ, Ferrario S, Busscher M, Angenent GC: Analysis of MADS box protein-protein interactions in living plant cells. Proc Natl Acad Sci USA 2002, 99:2416-242I.

27. Kato N, Pontier D, Lam E: Spectral profiling for the simultaneous observation of four distinct fluorescent proteins and detection of protein-protein interaction via fluorescence resonance energy transfer in tobacco leaf nuclei. Plant Physiol 2002, I29:931-942.

28. Shah K, Russinova E, Gadella TWJ, Willemse J, de Vries SC: The Arabidopsis kinase-associated protein phosphatase controls internalization of the somatic embryogenesis receptor kinase I. Genes Dev 2002, 16:1707-1720.

29. Bhat RA, Borst JW, Riehl M, Thompson RD: Interaction of maize Opaque-2 and the transcriptional co-activators GCN5 and ADA2, in the modulation of transcriptional activity. Plant Mol Biol 2004, 55:239-252
30. Vermeer JEM, Van Munster EB, Vischer NO, Gadella TWJ: Probing plasma membrane microdomains in cowpea protoplasts using lipidated GFP-fusion proteins and multimode FRET microscopy. J Microsc 2004, 2 | 4: 190-200.

31. Fujiwara MT, Nakamura A, Itoh R, Shimada Y, Yoshida S, Moller SG: Chloroplast division site placement requires dimerization of the ARCI I/AtMinDI protein in Arabidopsis. J Cell Sci 2004, I I 7:2399-2410.

32. Bhat RA, Miklis M, Schmelzer E, Schulze-Lefert P, Panstruga R: Recruitment and interaction dynamics of plant penetration resistance components in a plasma membrane microdomain. Proc Natl Acad Sci USA 2005, 102:3 135-40.

33. Elliott C, Müller J, Miklis M, Bhat RA, Schulze-Lefert P, Panstruga R: Conserved extracellular cysteine residues and cytoplasmic loop-loop interplay are required for functionality of the heptahelical MLO protein. Biochem J 2005, 385:243-254.

34. Seidel T, Golldack D, Dietz KJ: Mapping of C-termini of VATPase subunits by in vivo -FRET measurements. FEBS Lett 2005, 579:4374-4382.

35. Tonaco IAN, Borst JW, de Vries SC, Angenent GC, Immink RGH: In vivo imaging of MADS-box transcription factor interactions. J Exp Bot 2006, 57:33-42.

36. Feys BJ, Wiermer M, Bhat RA, Moisan LJ, Medina-Escobar N, Neu C, Cabral A, Parker JE: Arabidopsis SENESCENCE-ASSOCIATED GENEIOI stabilizes and signals within an ENHANCED DISEASE SUSCEPTIBILITYI complex in plant innate immunity. Plant Cell 2005, 17:260I-26I3.

37. Maple J, Aldridge C, Moller SG: Plastid division is mediated by combinatorial assembly of plastid division proteins. Plant $J$ 2005, 43:8II-823.

38. Miyawaki A, Llopis J, Heim R, McCaffery JM, Adams JA, Ikura M, Tsien $R Y$ : Fluorescent indicators for $\mathrm{Ca}^{2+}$ based on green fluorescent proteins and calmodulin. Nature 1997, 388:882-887.

39. Fehr M, Frommer WB, Lalonde S: Visualization of maltose uptake in living yeast cells by fluorescent nanosensors. Proc Natl Acad Sci USA 2002, 99:9846-985I.

40. Selvin PR: The renaissance of fluorescence resonance energy transfer. Nat Struct Biol 2000, 7:730-734.

4I. Clegg RM: Fluorescence resonance energy transfer. Curr Opin Biotechnol I995, 6: 103-II0.

42. Arai R, Ueda H, Kitayama A, Kamiya N, Nagamune T: Design of the linkers which effectively separate domains of a bifunctional fusion protein. Protein Eng 200I, 14:529-532.

43. Damelin M, Silver PA: In situ analysis of spatial relationships between proteins of the nuclear pore complex. Biophys J 2002 , 83:3626-3636.

44. He LS, Wu XL, Simone J, Hewgill D, Lipsky PE: Determination of tumor necrosis factor receptor-associated factor trimerization in living cells by CFP $\rightarrow>$ YFP $\rightarrow$ mRFP FRET detected by flow cytometry. Nucleic Acids Res 2005, 33:e6 I. I-I2

45. Lissandron V, Terrin A, Collini M, D'Alfonso L, Chirico G, Pantano S, Zaccolo M: Improvement of a FRET-based indicator for CAMP by linker design and stabilization of donor-acceptor interaction. J Mol Biol 2005, 354:546-555.

46. Sekar RB, Periasamy A: Fluorescence resonance energy transfer (FRET) microscopy imaging of live cell protein localizations. J Cell Biol 2003, 160:629-633.

47. Gordon GW, Berry G, Liang XH, Levine B, Herman B: Quantitative fluorescence resonance energy transfer measurements using fluorescence microscopy. Biophys J 1998, 74:2702-27I3.

48. Gadella TW Jr, van der Krogt GN, Bisseling T: GFP-based FRET microscopy in living plant cells. Trends Plant Sci 1999, 4:287-29I.

49. Berney C, Danuser G: FRET or no FRET: a quantitative comparison. Biophys J 2003, 84:3992-40I0.

50. Xia Z, Liu Y: Reliable and global measurement of fluorescence resonance energy transfer using fluorescence microscopes. Biophys / 200I, 81:2395-2402.

5I. Karpova TS, Baumann CT, He L, Wu X, Grammer A, Lipsky P, Hager GL, McNally JG: Fluorescence resonance energy transfer from cyan to yellow fluorescent protein detected by acceptor photobleaching using confocal microscopy and a single laser. J Microsc 2003, 209:56-70.

52. Valentin G, Verheggen C, Piolot T, Neel H, Coppey-Moisan M, Bertrand E: Photoconversion of YFP into a CFP-like species during acceptor photobleaching FRET experiments. Nat Methods 2005, 2:80I-80।. 
53. Becker W, Bergman A, Biskup C, Kelbaukas L, Zimmer T, Kloecker $\mathrm{N}$, Bendorf $\mathrm{K}$ : High resolution TCSPC lifetime imaging. Proc SPIE 2003, 4963: I-I0.

54. van Munster EB, Gadella TWJ: Fluorescence lifetime imaging microscopy (FLIM). Adv Biochem Eng Biotechnol 2005, 95: I 43-I75.

55. Pollok BA, Heim R: Using GFP in FRET-based applications. Trends Cell Biol 1999, 9:57-60.

56. Shaner NC, Steinbach PA, Tsien RY: A guide to choosing fluorescent proteins. Nat Methods 2005, 2:905-909.

57. Nguyen AW, Daugherty PS: Evolutionary optimization of fluorescent proteins for intracellular FRET. Nat Biotechnol 2005 , 23:355-60.

58. Rizzo MA, Springer GH, Granada B, Piston DW: An improved cyan fluorescent protein variant useful for FRET. Nat Biotechnol 2004, 22:445-449.

59. Shaner NC, Campbell RE, Steinbach PA, Giepmans BN, Palmer AE, Tsien RY: Improved monomeric red, orange and yellow fluorescent proteins derived from Discosoma sp. red fluorescent protein. Nat Biotechnol 2004, 22:I567-I572.

60. Zapata-Hommer O, Griesbeck O: Efficiently folding and circularly permuted variants of the Sapphire mutant of GFP. BMC Biotechnol 2003, 3:5.

61. Zhang J, Campbell RE, Ting AY, Tsien RY: Creating new fluorescent probes for cell biology. Nature Rev Mol Cell Biol 2002, 3:906-918.

62. Zacharias DA, Violin JD, Newton AC, Tsien RY: Partitioning of lipid-modified monomeric GFPs into membrane microdomains of live cells. Science 2002, 296:913-916.

63. Abedi MR, Caponigro G, Kamb A: Green fluorescent protein as a scaffold for intracellular presentation of peptides. Nucleic Acids Res 1998, 26:623-630.

64. Baird GS, Zacharias DA, Tsien RY: Circular permutation and receptor insertion within green fluorescent proteins. Proc Natl Acad Sci USA 1999, 96: I I24I-I I 246.

65. Magliery TJ, Regan L: Reassembled GFP: detecting protein-protein interactions and protein expression patterns. In Methods Biochem Anal Volume 47. Edited by: Chalfie M, Kain SR. Hoboken, New Jersey, USA: John Wiley \& Sons Ltd.; 2006:39I-405.

66. Ghosh I, Hamilton AD, Regan L: Antiparallel leucine zipperdirected protein reassembly: Application to the green fluorescent protein. J Am Chem Soc 2000, I 22:5658-5659.

67. Hu CD, Chinenov $Y$, Kerppola TK: Visualization of interactions among bZIP and Rel family proteins in living cells using bimolecular fluorescence complementation. Mol Cell 2002, 9:789-798.

68. Walter M, Chaban C, Schutze K, Batistic O, Weckermann K, Nake C Blazevic D, Grefen C, Schumacher K, Oecking C, Harter K, Kudla J: Visualization of protein interactions in living plant cells using bimolecular fluorescence complementation. Plant J 2004, 40:428-438.

69. Bracha-Drori K, Shichrur K, Katz A, Oliva M, Angelovici R, Yalovsky $S$, Ohad N: Detection of protein-protein interactions in plants using bimolecular fluorescence complementation. Plant J 2004, 40:419-427.

70. Tzfira T, Vaidya M, Citovsky V: Involvement of targeted proteolysis in plant genetic transformation by Agrobacterium. Nature 2004, 431 :87-92.

71. Diaz I, Martinez M, Isabel-LaMoneda I, Rubio-Somoza I, Carbonero P: The DOF protein, SAD, interacts with GAMYB in plant nuclei and activates transcription of endosperm-specific genes during barley seed development. Plant J 2005, 42:652-662.

72. Hackbusch J, Richter K, Müller J, Salamini F, Uhrig JF: A central role of Arabidopsis thaliana ovate family proteins in networking and subcellular localization of 3-aa loop extension homeodomain proteins. Proc Natl Acad Sci USA 2005, I 02:4908-49I2.

73. Lacroix $B$, Vaidya M, Tzfira T, Citovsky V: The VirE3 protein of Agrobacterium mimics a host cell function required for plant genetic transformation. EMBO J 2005, 24:428-437.

74. Li J, Krichevsky A, Vaidya M, Tzfira T, Citovsky V: Uncoupling of the functions of the Arabidopsis VIPI protein in transient and stable plant genetic transformation by Agrobacterium. Proc Natl Acad Sci USA 2005, I 02:5733-5738.

75. Loyter A, Rosenbluh J, Zakai N, Li J, Kozlovsky SV, Tzfira T, Citovsky $\mathrm{V}$ : The plant VirE2 interacting protein I. a molecular link between the Agrobacterium T-complex and the host cell chromatin? Plant Physiol 2005, I38: |3 |8-I32 I.

76. Stolpe T, Susslin C, Marrocco K, Nick P, Kretsch T, Kircher S: In planta analysis of protein-protein interactions related to light signaling by bimolecular fluorescence complementation. Protoplasma 2005, 226: 137-146.

77. Abe M, Kobayashi Y, Yamamoto S, Daimon Y, Yamaguchi A, Ikeda $Y$, Ichinoki $H$, Notaguchi $M$, Goto K, Araki T: FD, a bZIP protein mediating signals from the floral pathway integrator $F T$ at the shoot apex. Science 2005, 309:1052-1056.

78. Shimizu H, Sato K, Berberich T, Miyazaki A, Ozaki R, Imai R, Kusano $T$ : LIPI9, a basic region leucine zipper protein, is a fos-like molecular switch in the cold signaling of rice plants. Plant Cell Physiol 2005, 46: 1623-1634

79. Cole M, Nolte C, Werr W: Nuclear import of the transcription factor SHOOT MERISTEMLESS depends on heterodimerization with BLH proteins expressed in discrete sub-domains of the shoot apical meristem of Arabidopsis thaliana. Nucleic Acids Res 2006, 34: I28I-1292.

80. Zamyatnin AA, Solovyev AG, Bozhkov PV, Valkonen JPT, Morozov SY, Savenkov El: Assessment of the integral membrane protein topology in living cells. Plant J 2006, 46: I 45-I 54

81. Magliery TJ, Wilson CG, Pan W, Mishler D, Ghosh I, Hamilton AD, Regan L: Detecting protein-protein interactions with a green fluorescent protein fragment reassembly trap: scope and mechanism. J Am Chem Soc 2005, I 27:|46-I57.

82. Shyu YJ, Liu H, Deng X, Hu CD: Identification of new fluorescent protein fragments for bimolecular fluorescence complementation analysis under physiological conditions. Biotechniques 2006, 40:61-66.

83. Ozalp C, Szczesna-Skorupa E, Kemper B: Bimolecular fluorescence complementation analysis of cytochrome p450 2c2, $2 \mathrm{el}$, and NADPH-cytochrome $\mathbf{4 5 0}$ reductase molecular interactions in living cells. Drug Metab Dispos 2005, 33:1382-1390.

84. Remy I, Michnick SW: A cDNA library functional screening strategy based on fluorescent protein complementation assays to identify novel components of signaling pathways. Methods 2004, 32:381-388.

85. Hu CD, Kerppola TK: Simultaneous visualization of multiple protein interactions in living cells using multicolor fluorescence complementation analysis. Nat Biotechnol 2003, 2I:539-545.

86. Galperin E, Verkhusha V, Sorkin A: Three-chromophore FRET microscopy to analyze multiprotein interactions in living cells. Nat Methods 2004, I:209-2 I7.

87. Haustein E, Schwille P: Single-molecule spectroscopic methods. Curr Opin Struct Biol 2004, | 4:53 I-540.

88. Bacia K, Kim SA, Schwille P: Fluorescence cross-correlation spectroscopy in living cells. Nat Methods 2006, 3:83-89.

89. Digman MA, Brown CM, Sengupta P, Wiseman PW, Horwitz AR, Gratton E: Measuring fast dynamics in solutions and cells with a laser scanning microscope. Biophys / 2005, 89: I3 |7-I327.

90. Kim MC, Panstruga R, Elliott C, Müller J, Devoto A, Yoon HW, Park HC, Cho MJ, Schulze-Lefert P: Calmodulin interacts with MLO protein to regulate defence against mildew in barley. Nature 2002, 4 I 6:447-450

91. Schulze-Lefert P: Plant immunity: The origami of receptor activation. Curr Biol 2004, I 4:R22-R24 